Sept. 30th.-Temperature normal, pulse 72 ; mouth opened to the extent of half an inch. No pain, no risus, or spasm about the body. An enema of fifteen grains of chloral was given, and later in the day forty grains of compound jalap powder, which moved his bowels several times in the night and once on the following morning. On Oct. 1st temperature and pulse normal, stiffness of mouth greater; opening to the extent of a quarter of an inch. To have thirty grains of chloral every four hours in enema. On the 2nd trismus not diminished, pain in the back of neck, temperature $100^{\circ}$, pulse 98 . On the 3rd pulse 116 , temperature $99^{\circ}$; trismus increased, stiffness of neck greater; corners of mouth drawn down. Ordered forty grains of bromide of potassium every four hours, and forty grains of chloral as an enema every four hours. Evening: Pulse 128, very weak and compressible; frequently recurring spasms, followed by vomiting. Chloral omitted on account of the state of the pulse, and one drachm of bromide of potassium ordered every four hours. To have a bath of $100^{\circ} \mathrm{F}$. for an hour. Three teaspoonfuls of Brand's beef essence every second hour were easily swallowed without exciting spasm. On the 4th swallowed the medicine about six times; he thinking that ice stopped the spasm, he asked for it. Temperature $101 \cdot 3^{\circ}$. Twenty grains of chloral hydrate at once, and followed by twenty grains in three hours-if necessary subcutaneously. 11.30 $\mathrm{P}_{1} \mathrm{M}_{2}$ : Twelve grains given subcutaneously, and repeated at 12.30. Spasms as frequent as before, though patient felt drowsy. 4.30 A.M.: Twelve grains repeated subcutaneously; spasm less frequent and less severe ; patient very drowsy. Temperature, 2 A.M., $99^{\circ}$. On the 5th the injection of chloral was reveated at 8.30 and 12. Temperature 100 , pulse very feeble and compressible. Ordered twelve grains of chloral hydrate, sixty grains of bromide of potassium, and twenty drops of tincture of digitalis every four hours as an enema. Pulse so feeble that after the first dose the chloral was omitted. No spasm after 10.30, but the patient died exhausted, Oct. 6th. Not more than half the above enema could be retained at one time, the rectum being very irritable.

Remarks. - The patient was a strong healthy-looking man, and the symptoms were so slight that some hopes of his recovery were entertained. Dr. Wm. Carter also saw the case from the commencement.

CASE 2.-M. L- aged twenty-five, was admitted on Sept. 26th. He was a healthy young man, employed as a tramcar driver. While trying to whip some boys clinging to the car he slipped, and the car passed over his right leg. When admitted it was severely lacerated and bleeding fast, apparently from one of the tibial arteries. Esmarch's tube was at once put on by the house-surgeon, and within half an hour Mr. Ransford saw the patient and amputated at the seat of selection by lateral skin flaps and circular division of muscles. Hæmorrhage was rather free at the time and somewhat difficult to stop. The operation was done under the spray and dressed with the carbolic gauze. About two honrs afterwards rather sharp capillary hæmorrhage took place, necessitating the taking down of the stump. This was done under a large carbolic spray from a steam spray-producer. No vessel was found, but rather general cozing, which mostly ceased on exposure and clearing out clots, and elevating the stump. The dressings were reapplied, and the house-surgeon was directed to give a subcutaneous injection of three grains of ergotine if there were any fresh signs of the hæmorrhage. This was done in about an hour, when some blood showed through the dressing, but did not increase after this. The next day (Sept. 27th) the temperature was $100^{\circ}$ in the moring, and $101^{\circ}$ in the evening; pulse 100 , very compressible. The wound was dressed, and looked very well. After this the pulse and temperature remained nearly normal up to Oct. 3rd, when the temperature was $100^{\circ} 6^{\circ}$, and remained $99^{\circ}$ or $100^{\circ}$ up to Oct. 6 th, the morning on which he died, when it reached $103^{\circ}$. The leg was dressed every other day, nothing but a little serum being on the dressings after the first day.

On October 3rd, the day on which his temperature rose to $100^{\circ} \mathrm{F}$., he complained of his throat being sore. Pulse 74 , compressible. Nothing was seen in his throat; no risus; no trismus. Appetite good, able to take full diet. Next day he had dinner and breakfast, and took them without complaint. The first sign of stiffiness of the mouth was noticed in the morning at 1 A.M., and in the evening of the same day he had marked difficulty in opening his mouth and spasm in taking food. Had a free purge, followed by thirty grains of chloral hydrate every third hour in the form of an enema, to this was added bromide of potassium and digitalis, to avoid if possible the depressing effects of chloral. On the 5th the trismus had gradually increase, spasm appeared at every attempt to swallow, and food as well as medicine had to be given per rectum. On the following day the spasm was severe and long, the patient was hardly conscious ; bathed in cold sweat. Rectum very irritable, neither food nor medicine being retained. On the 6th morning temperature $100.4^{\circ}$; just before death $103^{\circ}$.

Remarks. - This case ran a very rapid course, and was apparently uninfluenced by chloral in any way. The tem. perature and dressing proved that there was no septic absorption, so that this would lead to prove that it cannot have anything to do with tetanus, as some think. The amputation was well above the lacerated tissues, so that the tetanus was most unexpected. It seemed as though he was going to get rapidly well. When he complained on the fourth day, at first it was hoped that imagination might have something to do with it, as he had heard of the first case ; but by the evening all doubt as to its nature was at an end, and chloral was at once given. At the necropsy about half a drachm of pus was found in a small abscess in the stump. Some might say this proved it not to be aseotic, but it was perfectly aseptic; still it was not suspected during life. I have had lately two other similar cases, but have no notes by me now.

\section{SOUTHPORT INFIRMARY.}

FATAL CASE OF DEPRESSED FRACTURE OF SKULL, WITH SLIGHT SYMPTOMS.

(Under the care of Dr. McNicoLL.)

For the following notes we are indebted to Mr. A. J. Popert, house-surgeon.

On the evening of Nov. 11th last, in a drunken brawl, the patient, a woman aged forty, received a blow on the head from a small kitchen poker. She was not stunned, and thought little of it; her friends easily stopped the slight hemorrhage that occurred from the small scalp-wound caused by the blow, and it was not until the morning of the 16 th that she applied at the infirmary. She complained of soreness of the scalp. Upon examination a small sloughy wound, large enough to admit the top of the little finger, was found slightly to the left of the median line of frontal bone, and close to its upper border. The bone was denuded of periosteum. She was advised to enter the infirmary, which she did next day. A more careful examination then revealed the fact that an almost circular piece of bone, about the size of a sixpence, immediately beneath the wound, was slightly depressed. The depression, however, was so slight as to be more evident to the touch than to the eye. It was entirely surrounded by a faint line of fracture, but was not starred, and was quite immovable. Pressure on this spot caused no pain. The patient complained of slight frontal headache and nothing else. The pupils were normal, responding freely to light. Her temperature was $100^{\circ}$; pulse 90. Patient had not shivered.

On the 18th a consultation was held as to the expediency of trephining, and taking into consideration the fact that five days had elapsed since the receipt of the injury, and that the patient presented no symptoms beyond headache of an intermittent character, and a temperature of $101^{\circ}$, it was decided to wait.

Next day the evening temperature was $103^{\circ}$, and slight diarrhœa set in. This was checked by astringents, and the temperature again fell to $101^{\circ}$. From this time till Dec. 3rd patient's condition remained unchanged. She slept well, took her liquid food with good appetite, and except for the headache was fairly comfortable; but on the evening of this day she became rapidly comatose, and died in that condition about twenty-four hours after.

At the necropsy the exterial aspect of the cranial rault presented the appearances already described. On removing the skull-cap a comminuted fracture of the inner table was found; it was circular and rather larger than the corresponding depression of the external table, and consisted of three segments, the peripheral borders of all of which were free and sharp. The dura mater was bathed in creamy yellow pus, and on cutting this membrane several ounees of purnlent fluid escaped. The whole of the right anterior 
lobe of cerebrum was disorganised, consisting of a dirtybrown semi-fluid mass; the cortex of the rest of this hemisphere showed signs of compression, but the left half brain seemed to be quite normal and healthy, nor had any pus found its way on this side of the head. All other organs were found to be healthy.

Remarks. - In this case the symptoms during life bore no proportion to the severity of the lesion, at no time did the patient present any of the clinical indications of acute meningitis; in fact, the only evidence of any mischief beyond the fracture were the headache and temperature; this fact alone makes the case of sufficient importance to record, and the question of trephining makes it additionally interesting.

\section{attedical Suriettis.}

\section{ROYAL MEDICAL \& CHIRURGICAL SOCIETY.}

The Condition of Large Arteries after Ligature under Antiseptic and Non-antiseptic Mecisures.-Axillary Aneurism treated by Antiseptic Ligature of third part of Subclavian Artery; Farticul Restoration of Lumen of Vessel.

THE ordinary meeting of this Society was held on the 8th inst., J. E. Erichsen, Esq., F.R.S., President, in the chair. Two papers illustrative of the inefficiency of the catgut ligature to produce permanent occlusion of arteries tied in their continuity were read, the one being contributed by Mr. Treves, and the other by Mr. M'Carthy. The main contention was that under antiseptics the changes around the vessels are too slight and temporary to aid in the occluding process.

A paper was read on "A Case illustrating the Condition of Large Arteries after Ligature under Antiseptic and Nonantiseptic Measures," by Mr. FredERICK TREVES, of which the following is an abstract:-The specimens upon which the paper is founded consist-together with other partsof the right carotid and subclavian arteries from a man, aged forty-seven, in whom those vessels had been ligatured for innominate aneurism. The patient gave a history of previous good health, and no definite date for the com. mencement of the local symptoms. A small aneurism of the innominate artery was diagnosed and ligature of its two branches resolved upon. On June 30 th, 1880 , the right carotid was ligatured above the omohyoid by Mr. James Adams, under whose care the patient was. The operation was performed under antiseptic precautions without hindrance or difficulty of any lind, a stout catgut ligature was used, and tied in a double and then a single hitch. The wound healed rapidly by first intention without the formation of a drop of pus, and in twelve days the antiseptic dressings were discontinued. After the operation the aneurism, although it did not diminish, ceased to increase. On July 21st, 1880, Mr. Treves ligatured the third part of the subclavian, the operation was also performed antiseptically, and the vessel was readily found; a stout catgut ligature was used and was tied in two simple hitches. During the night after the operation the patient loosened the antiseptic dressings and exposed the wound. On the evening of the next day he had a chill, the temperature rose to $1016^{\circ}$, and the edges of the wound were red and swollen. The wounds began to suppurate very freely; the stitches had to be removed to allow of the freer discharge of pus, and in thirty-six days the incision was almost healed, having closed up from the bottom. The carotid incision may fairly be regarded as a type of healing underantiseptic measures, and the subclavian incision as an open suppurating wound. After the subclavian was tied the aneurism improved greatly, all the previous symptoms of discomfort gradually disappeared, and the man was discharged thirty-six days after the operation with the tumour apparently cured, or at least greatly benefited. He returned, however, on Oct. 14th, 1880, complaining of severe pain in the back, of cough, and of general feebleness, and was readmitted. After a slight attack of hæmoptysis the patient died suddenly two days after admission. At the time of death, therefore, 108 days had elapsed since the carotid was ligatured, and eighty-seven days since the subclavian operation. Death'was found to be due to the rupture of an aneurism of the thoracic aorta ; this had produced great destruction of some of the dorsal vertebræ. The great vessels were examined in situ. A small aneurism about the size of a Spanish chestnut was found connected with the innominate artery at its commencement; its sac was almost filled with laminated fibrin, leaving only a cavity about the size of a horse-bean in the centre of the tumour, and that communicated with the vessel. The brachiocephalic vessel was quite patent, as were also the first portions at least of its two branches. In the region of the carotid operation the restoration of the parts to their normal condition was remarkable, the skin scar was slight and had formed no adhesions to the parts beneath, no trace of wound could be detected in the carotid sheath, no adhesions had formed between the artery and the vein, and every trace of the ligature had disappeared. No constriction could be seen in the vessel, and its external outline was unaltered and the seat of ligature indicated only by a faint transverse bluish mark on the vessel, due apparently to the thinness of the arterial coats in that situation. The vessel was found to be patent, but to be narrowed at the seat of ligature by a kind of perforated diaphragm or a miniature pylorus, leaving a central aperture about a line and a half in diameter. This diaphragm was formed by the incurvings of the ruptured ends of the middle coat, and as the external coat retained unaltered the external outline of the vessel, a minute triangular interval was thus formed, having its base represented only by the isolated external coat, and indicated by the bluish line, and its apex by the margin of the diaphragm, where the two cut ends of the middle coat met. As the two portions of the middle coat were only united to one another by the scantiest of connective tissues, the artery was very weak at the seat of ligature, and allowed of con. siderable angular bendings. The third part of the subclavian artery was found embedded in a mass of very firm inflammatory material, and was occluded for three-quarters of an inch, and entirely obliterated for half an inch. No trace of the ligature could be detected; the subclavian vein was patent. The aorta was very atheromatous, and the summit of its arch calcareons; the aperture leading from the aorta to the left carotid was extremely small, and that vessel was found to be occluded, save for a minute channel, through which air could only with some difficulty be forced. This unsuspected closure of the left carotid was evidently present at the time of the patient's admission, and much embarrassed the early diagnosis; for when the pabient came under notice it was observed that the left temporal pulse was much feebler than the right, that the aneurismal bruit (most clearly marked over the innominate tumour) was more distinct in the left than in the right carotid, and that in the left vessel a thrill conld be felt not noticeable in the right. These symptoms aroused strong suspicions of aneuism of the aortic areb, fand are explained by the almost solid condition of the left carotid. As an operation for closing a bloodvessel the carotid operation must be considered to have failed, snd the reasons of that fallure are matters of great importivee. In reviewing the subject $\mathrm{Mr}$. Treves deals in detail with the comparative physiological condition of the two vessels at the time of ligature, and urges that in this respect the condition of the subclavian artery at the time of ligature was actually less favourable than was that of the carotid when subjected to operation. In the details of the two operations and in the health and general surroundings of the patient, no disterences are apparent that could account for the differences in result. The conclusion, therefore, would appear to follow that the failure of the carotid ligature was dne to the antiseptic measures adopted. It would appear that these measures might almost err on the side of perfection, by producing too ready and too complete a return of the parts to their con dition previous to disturbance. Anatomically the healing of the wound was perfect, but would seem to have been too perfect for surgical purposes. A little so-called reaction in the wound, and a little of that firm inflammatory material almost too boun rifully supplied about the subclavian incision, would probably have effected the permanent closure of the artery. l'he comparative risks, however, attencing the healing of the two wounds are to be considered. The question remains as to whether, for some surgical purposes, the perfect healing aimed at by the supporters of the antiseptic treatment is desirable or successful.

The following is an abstract of the paper on "Aneurism of the Axillary Artery, probably ruptured; treated by Antiseptic Ligature of third part of Subclavian Artery; Partial 